\title{
How Bureaucracy Can Foster Burnout
}

\author{
Richard B. Gunderman, MD, $\mathrm{PhD}^{\mathrm{a}}$
}

James W. Lynch, MD

a. Department of Radiology, Indiana University School of Medicine, Indianapolis, Indiana

b. Department of Medicine, College of Medicine, University of Florida, Gainesville, Florida

Although it is not typically a focus in discussions of burnout, it is our contention that the increasing bureaucratization of contemporary medical practice and health care is one of the most important sources of emotional exhaustion, depersonalization, and inefficacy confronting health professionals of all types, including radiologists, radiologic technologists, and other radiology personnel. Although bureaucracy can enhance organization and impartiality, it often exacts too high a human price from patients and health professionals.

Sociologist Max Weber [1] described bureaucracy as the most rational system for organizing large-scale human activity. He based this assessment on bureaucracy's ability to establish and maintain order, its strong distaste for inefficiency, and its tendency to constrain the role of personal preferences. However, Weber was well aware of bureaucracy's potential to undermine the freedom, creativity, and distinctive personalities of individuals, and he likened the effect of life in bureaucracy to existence in a purely rational "iron cage" that values predictability and control over all else.

\section{Standardization First}

Most bureaucracies fall short of Weber's ideal. Because decision making is often highly centralized, meaning that those making choices for an organization are far removed from the human encounters that 
define them, bureaucracies force frontline professionals either to suspend ethical and professional aspirations for the sake of following the rules or to ignore certain rules and develop workarounds to ensure that patients get the care they need. Too often, bureaucracies value adherence to rules more highly than pursuit of mission.

In one case, a health care system (whose name is not disclosed for fear of retribution) adopted an accountable payment model that required assessment of pain and emotional distress. When an internal audit indicated that fewer than half of a medical practice's health care records documented such care, the health care organization added a line to the medical record template that stated simply that both had been evaluated and addressed during each visit. As a result, compliance soared to more than $90 \%$. Yet many of the health professionals involved reported that they continued to perform such assessments only when medically indicated.

Such a case illustrates how a seemingly highly rational organizational form can in fact promote a culture of irrational inconsistency. Health professionals judge that the costs associated with noncompliance so outweigh the benefit of defending the integrity of the medical record that they accept such changes, on the presumption that those who really need to know — other health professionals — will discern which parts of the record they can truly rely on. Over time, as more and more such compliance-based compromises take place, cynicism and even mendacity begin creeping upward.

As a rational form of organization, bureaucracy places a substantial premium on consistency and coherence. Its most ardent partisans assume that there is one right way of doing everything, and people who deviate from prescribed policies and procedures must be guilty of error. This creates an insidious but powerful bias toward the development of a compliance culture in which a relatively few people at the top impose their vision of everyone else, defining their own success to a large extent by their ability to make everyone else in the organization toe the line.

\section{Depersonalization}


The problem, of course, is that all patients and health professionals who seem to fit into a particular category are not identical to each other. For example, a patient with both chronic obstructive pulmonary disease and heart failure was assigned by a member of the triage team to the heart failure pathway, which resulted in a delay in providing treatment for chronic obstructive pulmonary disease, which ultimately turned out to be the most important contributor to the patient's respiratory distress. On the basis of the heart failure flowchart, the care was perfect, but in terms of the patient's medical needs, care was both ineffective and inefficient.

When it comes to decision making, institutionally defined categories can assume greater importance in management and policymaking than the medical needs of the patient. The bureaucracy evaluates itself on the basis of abstract categories, whereas health professionals evaluate themselves and one another on the basis of the needs of particular patients. Again, the dissonance between serving the policies and procedures of a bureaucracy and serving the patient the health professional is facing can become intolerable, resulting in demoralization.

Bureaucracies are inherently impersonal, whereas providing good care to patients is an inherently personal endeavor. Bureaucracies always move toward depersonalization, in large part because that is exactly what they are designed to do. They want to assign everyone to a category or position, not to recognize them as distinct individuals. Yet when it comes to treating patients, particularly medically complex ones, patients may overlap multiple categories in ways that make it very difficult for a health professional to treat them as if they were all the same.

Adherence to standards is no guarantee that a patient will not suffer. An audit of a hospice's medical records disclosed that a substantial number of patients lacked sufficient documentation demonstrating the need for such services, resulting in financial penalties. Detailed checklists were introduced, with timeconsuming documentation requirements. After one 48-hour delay in establishing hospice eligibility, a nurse (who requested anonymity) arriving at a patient's home was greeted by a family angry that the 
patient had died in the interim. The institution had satisfied its requirements, but a patient went uncared for.

\section{Policies and People}

Put another way, the problem is a decoupling of formal organizational policies and procedures from the ways in which health professionals actually do their work. Many health professionals feel a greater sense of accountability to patients and families, as well as to the vision of excellence esteemed by their profession, than to any institution's policies and procedures. From their point of view, it does little good to adhere to standards if doing so does not serve the interests of a patient, and ignoring or fudging such standards is not too high a price to pay to put patients first.

Consider another example, this time drawn from the world of medical education. A medical school (the name of which is not disclosed to avoid possible retribution) identified a problem with tardiness in grade submission. The school proclaimed that clerkship directors would be held accountable if all evaluations were not submitted on time, despite the fact that they often had to collate reports from many sites and preceptors. Over time, grade submission times declined. However, the quality of the evaluations declined at an equal or even faster rate, such that generic comments tended to replace distinctive insights into each student's work.

If the purpose of requiring teachers to submit grades is to prove that such evaluations can be submitted in a timely fashion, then increasing penalties for late submissions proved an effective policy. If, however, the purpose of submitting evaluations is to gain meaningful insight into students and their work, including the identification of distinctive strengths, weaknesses, and recommendations for improvement, then the new grade policy proved an abject failure. Unfortunately, such changes tend to benefit those highest in the organization, while discouraging those on the front lines.

\section{The View From the Top}


In some cases, the conflict between what is good for those at the top of an organization and those actually doing the organization's daily work can be so great that one or the other finds the situation intolerable. This situation can be further exacerbated when one group regards the other as operating in bad faith. In some circumstances, health professionals may have reason to think that some of the people in the upper echelons of health care organizations spend more time managing their image than they do attending to the needs of patients and those who care for them.

For example, a new surgical practice manager was hired with a mandate to increase the rate at which each surgeon generated clinical revenue. A surgeon (who requested anonymity) with a deep professional commitment to knowing each of her patients well was identified as an outlier. The practice manager insisted that she spend only as much time with each patient as absolutely needed and no more. Faced with such a demand, the surgeon retired, to the great distress of her colleagues. Yet at year's end, the manager was rewarded with a handsome bonus.

Bureaucracy has a natural preference for quantitative measures, which seem to make it easier to compare different health professionals, practices, and hospitals and health care organizations. They naturally tend to introduce standardized surveys and metrics that treat work and the people who do it in standardized form. Although this bears at least a patina of fairness and objectivity, what it frequently does is undervalue or even ignore special contributions made by each health professional. And depriving professionals the opportunity to tell their story is particularly discouraging to the best among them.

\section{Going Forward}

The point of this analysis is not to label bureaucracy as an inherently evil form of organizing health care. To some degree, bureaucracy has an important role to play. However, bureaucracies by their very nature exist in tension with the aspirations and codes of ethics of health professionals, and if this tension is not well understood, it can degenerate into conflict and cynicism, eventuating in high rates of burnout. To 
avoid this fate, health professionals need to understand bureaucracy at least well enough to be on the look out for and counteract some of its most pernicious tendencies. 


\section{Reference}

Weber M. Bureaucracy. In: Waters T, Waters D, trans, eds. Weber's rationalism and modern society. New York: Palgrave-Macmillan; 2005. 